\title{
Conductance relaxation in Langmuir-Blodgett manganese phthalocyanine (PcMn) films in inhomogeneous electrical field
}

\author{
Z. Kazantseva', V. Kislyuk ${ }^{2}$, I. Kozyarevych ${ }^{2}$, V. Lozovski ${ }^{1,2}$, O. Tretyak ${ }^{2}$ \\ ${ }^{1}$ Institute of Semiconductor Physics, NAS of Ukraine, \\ 45, prospect Nauky, 03028 Kyiv, Ukraine; phone/fax: (+380) 445255530 \\ ${ }^{2}$ Taras Shevchenko Kyiv National University, Radiophysics Department, \\ 2 build 5, prospect Academician Glushkov, 03022 Kyiv, Ukraine \\ Corresponding author: Igor Kozyarevych, e-mail: kio@univ.kiev.ua
}

\begin{abstract}
The conductance relaxation of Langmuir-Blodgett films of manganese phthalocyanine in inhomogeneous electrical field was studied. Inhomogeneous electrical field was achieved by using the lateral surface of reverse-biased Si $p$ - $n$ junction. The conductance of new film increases up to saturation with the characteristic time about 10 hours. After that the film has been kept in air for a long time (about 50 days), on application of the back bias the conductance slowly decreased with the characteristic time more than 10 hours. These properties are associated with appearance or disappearance of the bonds between molecular stacks in the film.
\end{abstract}

Keywords: manganese phthalocyanine, Langmuir-Blodgett film, conductance, molecular stacks, silicon $p-n$ junction.

Manuscript received 23.09.05; accepted for publication 25.10.05.

\section{Introduction}

A great interest is being shown in thin organic films, because they are promising to be applied as materials for molecular electronics [1], in particular, as material for electroluminescent structures [2]. In this connection, nowadays the interest for studies of phthalocyanine films is arisen [3]. Phthalocyanine molecules have a specific geometrical structure (Fig. 1) that is a reason for their ability to form the metal-organic coordination compounds, the so-called metal-phthalocyanine (PcM). As a result, created are the molecular complex, in which a metal atom is located in the centre of the molecule. The metal atom and molecular frame form the so-called coordination bonds that allow the metal to have ability for binding the additional chemical bonds. This ability is the central point of the idea of this study.

Nanowires formed of TCNQ-TTF salts have been investigated for a long time as a prominent example of using the Langmuir-Blodgett (LB) technique to form nanoobjects $[4,5]$. The phthalocyanine molecules have the same properties. Namely, cofacially stacked macrocycles can also form nanowires. Fig. 1 shows an example of the macrocycle - a phthalocyanine molecule in which metal occupies the central position (the "M" designation in the structural formula in the left side of Fig. 1). The molecular stacks of phthalocyanines can be formed during the molecular crystal growth [6]. The same stacks are formed during preparation of monomolecular films with the LB technique that is well known [7-11]. Phthalocyanine films were studied as field-sensitive materials (i.e., interactions with applied electric, magnetic or electromagnetic fields) and as chemo-sensitive materials (i.e., interactions with other chemical species) [12-14]. The anomalous behavior of conductance of copper-phthalocyanine LB films was observed earlier [9, 11]. In particulary, the super-high lateral conductance increase was observed. Namely, the lateral conductance was more than that normal to the film surface by a factor of $10^{6}$. This conductance increase was related with the stack formation in the film $[7,9,11]$. The idea that the high lateral conductance is related with quasi-metal conductance along the monoatomic one-dimension metal wires inside the stacks can be fruitful for explanation of this phenomenon.

The behavior of lateral conductance in the LB tetratertbutyl-manganese phthalocyanine (PcMn) films was studied in this work. High sensitivity of the LB phthalocyanine films to electric field effect is expected. This work is devoted to the study of the inhomogeneous electrical field influence on the lateral conductance of PcMn LB films with the short hydrocarbon radicals $\left(\mathrm{R}=\mathrm{C}\left(\mathrm{CH}_{3}\right)_{3}\right)$. Molecules of these films are stacked. The central atoms of the molecules form monoatomic metal 

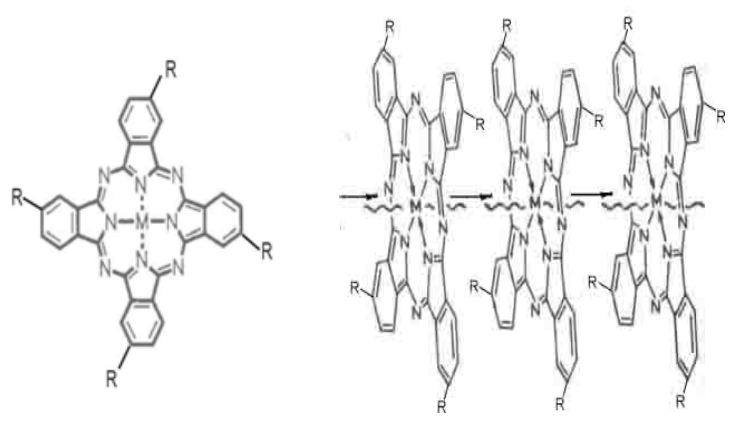

Fig. 1. Metal-substituted phthalocyanine molecule (left) and phthalocyanine molecules stacked cofacially in chain (right), $\mathrm{R}=\mathrm{C}\left(\mathrm{CH}_{3}\right)_{3}$.

wires. As a result, the film itself is in general nonconductive except local regions. The particular attention is given to the nanowire alignment. The nanowire alignment arises due to electrical field effect. This influence of homogeneous lateral electric field on the lateral conductance of PcCu LB films was observed in works $[7,9,11]$. A reverse-biased $p-n$ junction is the most appropriate substrate to study changes in conductance associated with alignment of the nanowires because there is inhomogeneous electrical field on the lateral surface.

\section{Experiment}

Multilayer PcMn films were deposited on lateral surfaces of silicon $p-n$ junctions by the LB technique. Silicon $p$-n junction is a handy substrate to investigate the high-resistance PcMn films because a current at reverse bias depends mainly on the conditions on the lateral surface of $p-n$ junction. A reverse-biased $p-n$ junction also provides an inhomogeneous electrical field. A silicon $p-n$ junction is just chosen because the electrophysical properties of phthalocyanines such as vanadium oxide phthalocyanine and copper phthalocyanine on silicon surface are known [7].

Before deposition of LB films, substrates were prepared. The lateral surfaces of the $p$ - $n$ junctions were polished with diamond paste for imparting the necessary shape and smooth surface to the substrates. The direction of the polishing was both parallel and perpendicular to the $p-n$ junction since influence of the polishing direction on the character of the conductance relaxation was not known. Then all substrates were degreased in boiling alcohol for about $20 \mathrm{~min}$. The removal of the thermal oxide in the boiling solution of the hydrogen peroxide and ammonia took about $10 \mathrm{~min}$. The etching of the silicon $p$ - $n$ junction substrates in $40 \%$ solution of $\left(\mathrm{NH}_{4}\right)_{2} \mathrm{Ti}_{2} \mathrm{~F}_{6}$ was made to prepare microscopically smooth surfaces.

PcMn LB films were prepared using a facility with the automatic system of the maintenance of the constant surface pressure inherent to the molecular monolayer on
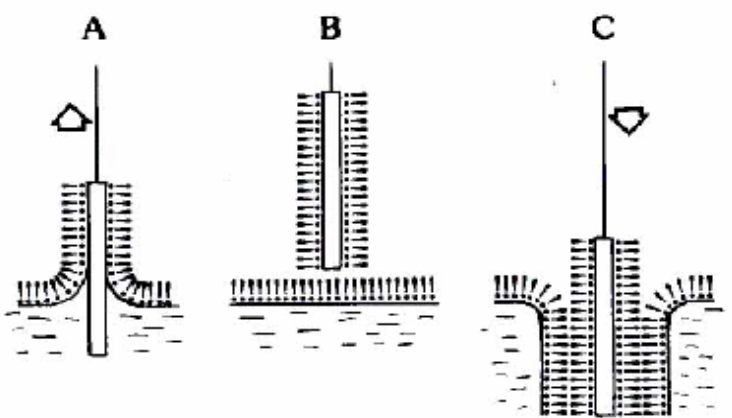

Fig. 2. Method of preparation of the LB films. One monolayer is deposited in series during unitary immersion.

the water surface. The scheme and construction of this device are described in [7]. The structural formula of the tetrasubstituted PcMn with substituting hydrocarbon radicals is shown in Fig. 1. The first stage of producing the LB film is formation of a monomolecular pellicle of PcMn on the water surface. Then the transfer of the monolayers was carried out onto the hydrophobic substrates at the room temperature and constant surface pressure by consecutive immersion and taking out these substrates that were preliminary cleaned with ultrasound in chloroform (Fig. 2). The molecules of PcMn are transferred onto the hydrophobic substrate because of the specific structure of molecules, which have hydrophilic and hydrophobic groups. Two-, four-, and ten-monolayer films were obtained on the lateral surfaces of the $p-n$ junctions.

Conductance relaxation of deposited PcMn monolayers was studied by applying a permanent back bias to the $p-n$ junction (Fig. 3 ) and the registration of changes in the current that flows through the series $p$ - $n$ junction and a load resistor $R(0.03 \%$ precision $)$. This current was determined from a voltage drop on the load resistor. Each sample was placed into the dark thermostat before measurements. There was a room temperature inside the thermostat.

\section{Results and discussion}

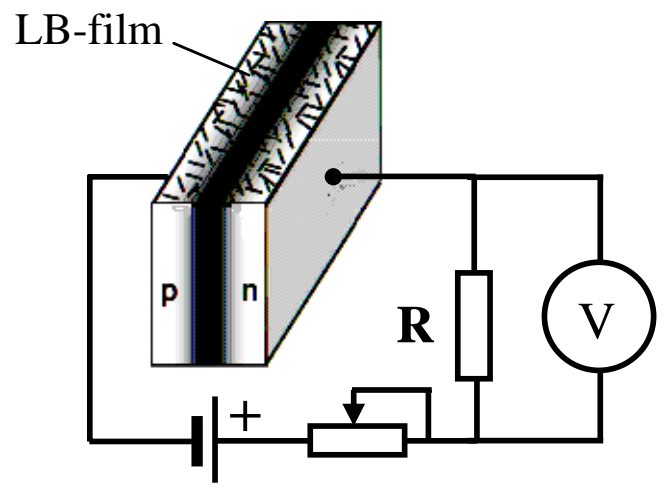

Fig. 3. Setup of the experiment. 


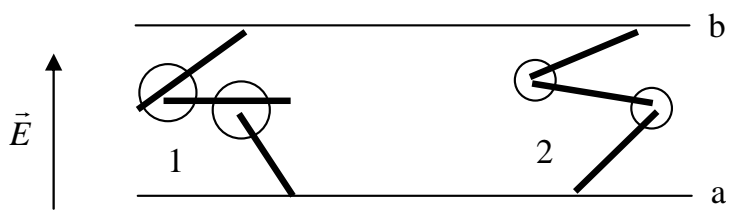

Fig. 4. For explanation of the conductance dependence on the structure of the film consisting of molecular stacks (marked as bold lines).

Results of our study of conductance relaxation are shown in Figs 5 and 6 . The unexpected results characterising the long-time current (conductance) relaxation can be explained as follows: as it was mentioned above, the structure of MnPc LB films is conceivably close-packed molecular stacks $[9,11]$. The conductance of these films is of a dielectric type with the exception of local regions - nanowires (or molecular assemblies) that are formed by atoms of manganese due to molecular stacking. Nanowire conductance is quasimetal. But internanowire conductance has hopping nature. It means that the number of the regions separating the beginnings and the ends of the nanowires defines the conducting properties of the film. Indeed, let the regions connecting the end of one molecular stack and beginning of the other one are characterized by the hopping conductivity $\sigma_{H}$ and the regions between arbitrary inter-stack contacts (except the end-beginning connections) $-\sigma_{S}$, and $\sigma_{H}>\sigma_{S}$. Therefore, the conductance between sections "a" and "b" along the right complex is much higher than that of the left one showed in Fig. 4. Here the molecular stack is designated as a bold line. The electrical field on the lateral surface of the $p-n$ junction is inhomogeneous. Therefore, the

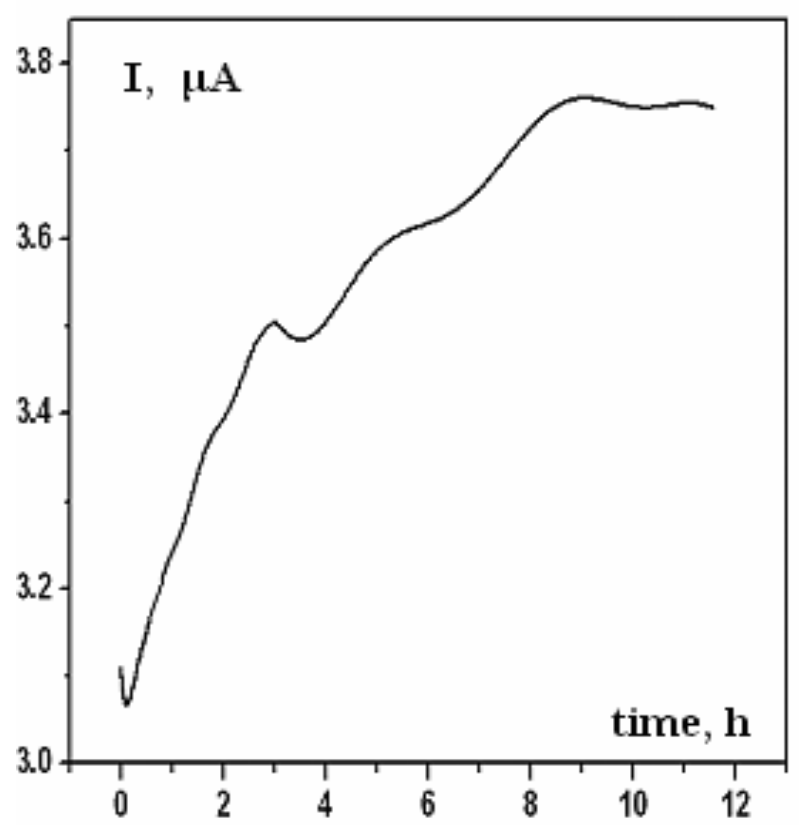

Fig. 5. The long-time current relaxation, two-layer sample, parallel direction of the polishing, the back bias is $2.74 \mathrm{~V}$. molecular assemblies are located in inhomogeneous electrical field. Let us begin with consideration of two molecular assemblies pinned by a weak binding force at the surface in homogeneous electrical field. A monoatomic needle located inside the phthalocyanine assembly is polarized by the action of the electrical field (i.e., the electron density on this metallic needle is shifted along the direction opposite to the field, and assembly acquires some considerable dipole moment). It is well known that the moment of rotation (shown by arrows in Fig. 7) $N=p E \sin \theta$ is applied to the dipoles that are turned through the angle $\theta$ to the electrical field, where $p$ is the dipole moment, $E$ is the electrical field. As shown in Fig. 7a, the molecular stack 2 prevents turning the molecular stack 1 , because the molecular stack 2 is acted upon by about the same magnitude of the moment of force as the molecular stack 1 but in the opposite direction. Therefore, molecular assemblies do not turn round, and conductance of the film keeps its value. Another process proceeds in the case of inhomogeneous filed. Indeed, in the presence of the nonzero gradients of the field, the force $\vec{F}=\vec{p} \cdot \operatorname{grad} \vec{E}$ acts on the molecular stack as a whole. This force can pick dipole from the pinning centre and shift it. If, for example, the force of the pinning of the first dipole is less than that of the second one, then the first dipole is shifted towards the field gradient (let do it towards the field direction) (Fig. 7b)) until fixation on another pinning centre will be reached. Then, both molecular complexes get the ability to turn (Fig. 7c)), and the conductance of this region can increase considerably. Molecular stacks will take up

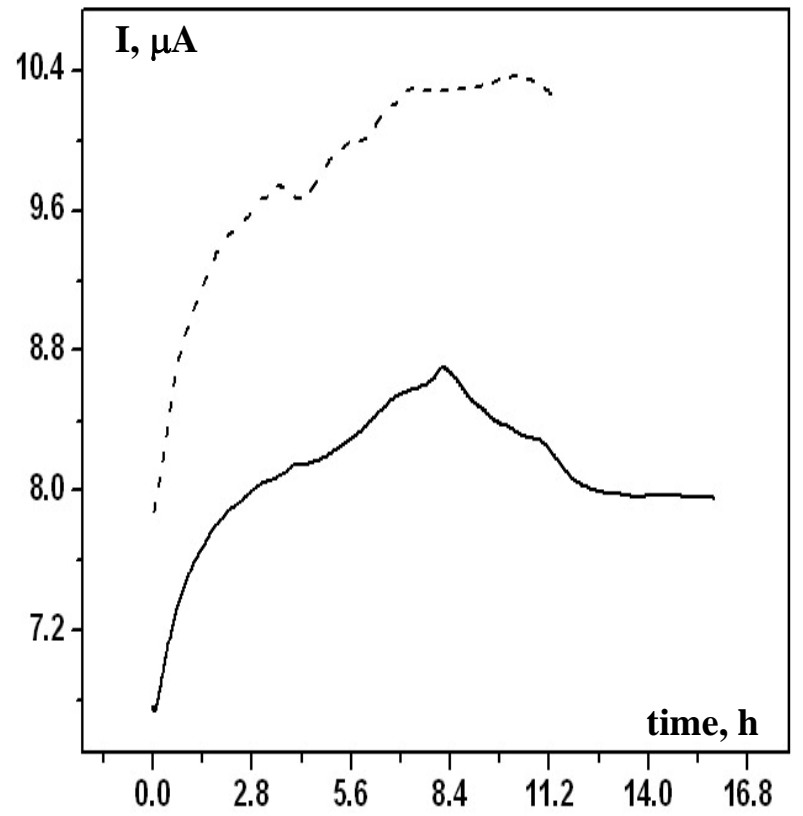

Fig. 6. The long-time current relaxation, ten-layer sample, parallel direction of the polishing, the back bias is $2.74 \mathrm{~V}$; dash line - the repetition of the measurement. 


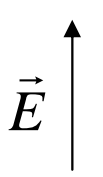

a)

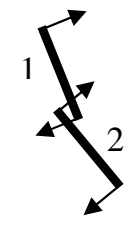

b)

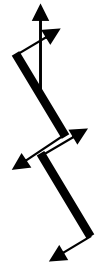

c)
Fig. 7. Moments and forces, acting upon the dipoles in homogeneous (a) and inhomogeneous (b) electric fields.

positions that are optimal for present electrical field, (i.e., the dipoles take a position corresponding to the minimum of the energy), and after 9-14 hours keeping the conductance of the film goes into saturation (see Figs 5 and 6). The conductance is reduced for the first 7 10 minutes because the distances between nanowires are largest.

The current slump was observed in the ten-layer film after keeping it for about eight hours (Fig. 6). This phenomenon can be explained as follows. After alignment of the stacks with preferred orientation along the field direction in the plane of each molecular layer, the processes of aligning in the planes perpendicular to the molecular layer plane begin to proceed (see Fig. 8b). Then, the electrical bonds between the stacks from different (but adjacent) layers are destroyed. As a result, the conductance of the film decreases when nanowires is aligned layer-by-layer because of the conductance between layers decreases.

The swing of some molecular stacks occurs during the voltage drop across the $p$ - $n$ junction for a long time. Repeated measurement shows conductance growth that begins from about that point which corresponds to the saturation on the previous curve.

The same results were obtained in samples that differed from the described above ones in the direction of polishing (i.e., with perpendicular to $p$-n junction

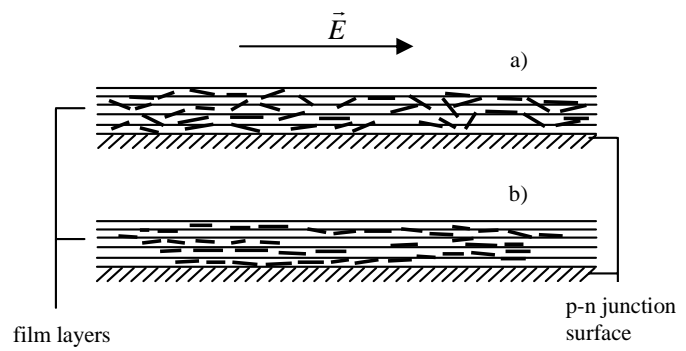

Fig. 8. a) weakly-ordered film (till 8 hours) that has a bigger conductance than (b) - layerwise aligned nanowires (after 8 hours) because of better conductivity between layers in case (a).

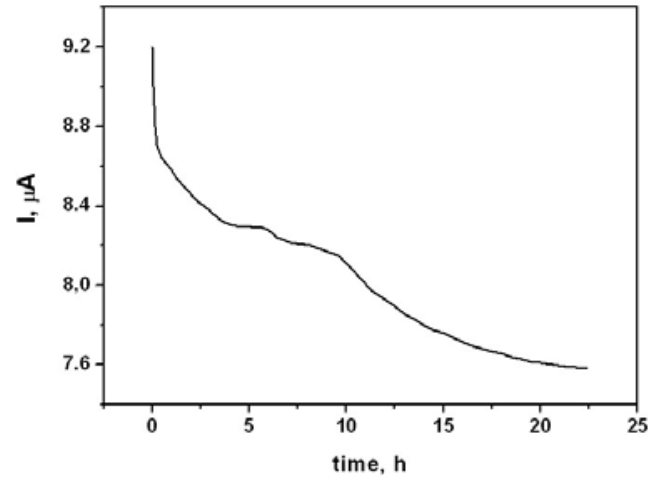

Fig. 9. The long-time current relaxation, the same sample as in Fig. 5, the back bias is $2.74 \mathrm{~V}$.

direction of polishing). The character of the current relaxation remained also without influences at back biases of $p$ - $n$ junctions equal to 5 and $10 \mathrm{~V}$.

As time goes by, there arose some bonds between stacks, and applying the back bias to the diode destroys these bonds by causing stack rotation, so conductance is decreased. The decreasing conductance, which can achieve up to 17.6 and $31.5 \%$, is shown in Figs 9 and 10 , respectively. It can be explained by the preliminary ordering of the sample shown in Fig. 9, across which the back bias was applied before. The results shown in Fig. 10 are concerned the sample that wasn't preliminary ordered by the electrical field, then destruction of bonds between molecular stacks result in decreasing the conductance. It should be noted that applying the direct bias across the field ordered film with heating simultaneously, leads to the behavior of the conductance like that in green films (see Fig. 11). Difference between dashed and solid lines in this figure arises because of insufficient duration of applying direct bias and heating for total misorientation of the stacks as well as breaking the bonds between it. Conductance relaxation was also studied in the films formed by molecules with long radicals. There was not observed this process in these

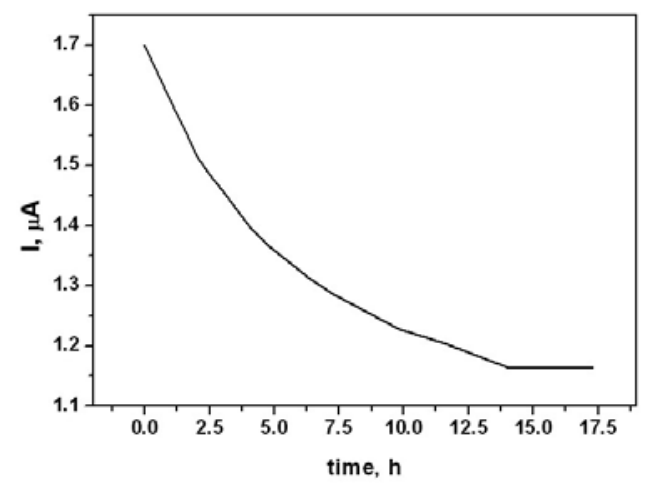

Fig. 10. The long-time current relaxation, non-measured sample, the back bias is $2.74 \mathrm{~V}$, the parallel direction of the polishing, four layers were deposited. 


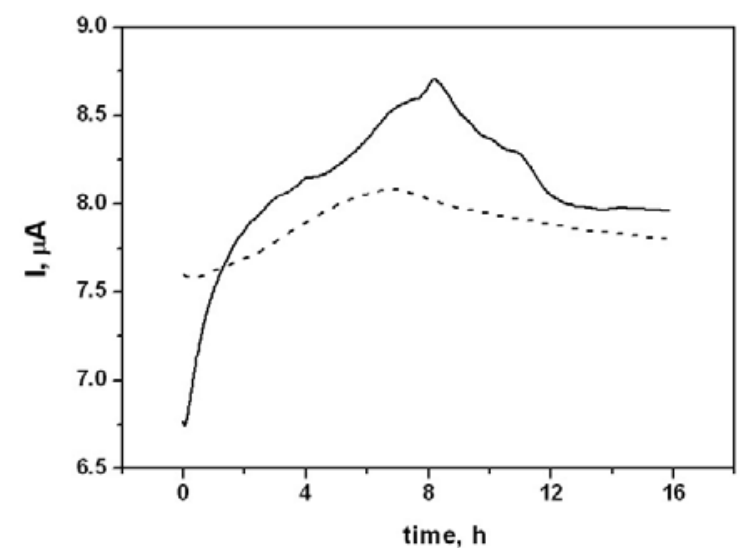

Fig. 11. The long-time current relaxation, solid line - the same sample as in Fig. 5, the back bias is $2.74 \mathrm{~V}$, dash line - repetition after heating at about $55^{\circ} \mathrm{C}$ and forward bias over 3 hours.

films. The behaviour of the back current through the $p$ - $n$ junction does not differ from that in the $p-n$ junction that uncovered by the film. Therefore, it was supposed that the molecules with long radicals do not form the stacks.

\section{Conclusions}

The relation between the structure and electrophysical properties of the tetrasubstituted PcMn LB films with substituting hydrocarbon radicals was ascertained. The long-time conductance relaxation was observed in PcMn LB films. This occurred because of the nonzero gradient of the electrical field. The electrical field shifts molecular stacks from the pinning centers, and then they can align along the field. Aligning molecular stacks increases the conductance. After 9-14 hours keeping, the film conductance goes into saturation. The theory of aligning the stacks is confirmed by similar experiments with molecules that have long tails and can't form the molecular stacks. Applying the direct bias across the field ordered film with heating simultaneously leads to the behavior of the conductance like that in new films.

\section{Acknowledgements}

Authors are very grateful to $\mathrm{K}$. Pokyd'ko and O. Pokyd'ko for their help in preparation of the samples and performing the measurements.

\section{References}

1. J.M. Tour, Molecular Electronics. World Scientific, London, Singapore (2003).

2. M. Pfeifer, K. Leo, X. Zhou et al., Doped organic semiconductors: Physics and application in light emitting diodes // Organic Electron. 4, p. 89-103 (2003).

3. N. Kobayashi, Phthalocyanines // Current opinion in solid state and materials science 4, p. 345-353 (1999).

4. T. Akutagawa, T. Ohta, T. Hasegawa, T. Nakamura, C.A. Christensen, J. Becher, Formation of oriented molecular nanowires on mica surface // Proc. Nat. Acad. Sci. USA 99, p. 5028-5033 (2002).

5. T. Ishiguro and K. Yamaji, Organic superconductors // Springer-Verlag Series in Solid-State Science $\mathbf{8 8}$ (1990).

6. Phthalocyanines. Properties and Applications, Ed. by C.C. Leznoff and A.B.P. Lever. VCH Publ., N.Y. (1989).

7. Z.I. Kazantseva, N.V. Lavrik, A.V. Nabok, B.A. Nesterenko, Yu.M. Shyrshov, The physical properties of the dielectric Langmuir-Blodgett films on silicic surface // Ukr. Fiz. Zhurn. 34, p. 1398-1404 (1989) (in Russian).

8. R.A. Hann, S.K. Gupta, J.R. Fryer, B.L. Eyers, Electrical and structural studies on copper tetra-tertbutyl phthalocyanine Langmuir-Blodgett films // Thin Solid Films 134, p.35-42 (1985).

9. Z.I. Kazantseva, N.V. Lavrik, A.V. Nabok, Conductance of the tetra-substituted vanadyl and copper phthalocyanine Langmuir - Blodgett films // Poverkhnost' 8, p.87-93 (1991) (in Russian).

10. S. Baker, M. C. Petty, G. G. Roberts, M. V. Twigg, The preparation and properties of stable metal-free phthalocyanine Langmuir-Blodgett films // Thin Solid Films 99, p.53-59 (1983).

11. B.A. Nesterenko, A.V. Nabok, Molecular ordered structures on solid surfaces // Theoretical and Experimental Chemistry, 29 (4), p. 291-305 (1993).

12. I. Yamazaki and N. Ohta, Photochemistry in LB films and its application to molecular switching devices // Pure \& Appl. Chem. 67 (1), p. 209-216 (1995).

13. A.V. Nabok, Z.I. Kazantseva, N.V. Lavrik, B.A. Nesterenko, Nitrogenoxide gas sensor based on tetratertbutyl copper phthalocyanine Langmuir-Blodgett films // Intern. J. Electronics 78 (1), p. 129-133 (1995).

14. T. Kato, N. Iwata and H. Yamamoto, Electrochromic properties of phthalocyanine derivative LangmuirBlodgett films // Advanced Nanomaterials and Nanodevices (IUMRS-ICEM 2002, Xi'an, China, 10-14 June, 2002, p.545-555 (2002). 